Pacific Northwest

National Laboratory

Operated by Battelle for the

U.S. Department of Energy

\title{
FY 2005 Laser Development Final Report
}

\author{
M. C. Phillips \\ T. L. Myers \\ M. S. Taubman
}

December 2005

Prepared for the U.S. Department of Energy under Contract DE-AC05-76RL01830 


\title{
DISCLAIMER
}

This report was prepared as an account of work sponsored by an agency of the United States Government. Neither the United States Government nor any agency thereof, nor Battelle Memorial Institute, nor any of their employees, makes any warranty, express or implied, or assumes any legal liability or responsibility for the accuracy, completeness, or usefulness of any information, apparatus, product, or process disclosed, or represents that its use would not infringe privately owned rights. Reference herein to any specific commercial product, process, or service by trade name, trademark, manufacturer, or otherwise does not necessarily constitute or imply its endorsement, recommendation, or favoring by the United States Government or any agency thereof, or Battelle Memorial Institute. The views and opinions of authors expressed herein do not necessarily state or reflect those of the United States Government or any agency thereof.

\author{
PACIFIC NORTHWEST NATIONAL LABORATORY \\ operated by \\ BATTELLE \\ for the \\ UNITED STATES DEPARTMENT OF ENERGY \\ under Contract DE-AC05-76RL01830
}

Printed in the United States of America
Available to DOE and DOE contractors from the Office of Scientific and Technical Information,
P.O. Box 62, Oak Ridge, TN 37831-0062;
ph: (865) 576-8401
fax: $(865)$ 576-5728
email: reports@adonis.osti.gov

\begin{abstract}
Available to the public from the National Technical Information Service, U.S. Department of Commerce, 5285 Port Royal Rd., Springfield, VA 22161 ph: (800) 553-6847 fax: $(703) 605-6900$ email: orders@ntis.fedworld.gov online ordering: http://www.ntis.gov/ordering.htm
\end{abstract}

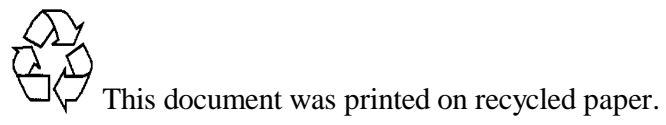




\title{
FY 2005 Laser Development Final Report
}

\author{
M. C. Phillips \\ T. L. Myers \\ M. S. Taubman
}

December 2005

Prepared for

the U.S. Department of Energy

under Contract DE-AC05-76RL01830

Pacific Northwest National Laboratory

Richland, Washington 99352 



\section{Summary}

The Laser Development Task of Pacific Northwest National Laboratory's (PNNL) Remote Spectroscopy project (PL211I) is focused on the development of novel laser technology for a new generation of standoff and in-situ chemical sensors for detecting the proliferation of nuclear weapons. These lasers will improve the sensitivity, flexibility, or range of active standoff sensors, enable ultra-trace in situ sensors with enhanced selectivity, as well as greatly improve calibration of passive standoff sensors. In particular, laser transmitters with minimal size, weight, and power consumption (SWAP) are needed to meet the requirements for a variety of in situ or short-range stand-off sensors and sensors for small UAVs or other platforms. These laser transmitters need to be rugged and free of requirements for consumables such as liquid nitrogen. Many sensing techniques also require lasers that produce a single narrow wavelength (single longitudinal mode). Lasers that provide high continuous-wave (CW) output power on a single line at operating temperatures accessible with thermoelectric (TE) cooling are therefore essential for sensor applications.

Quantum cascade (QC) lasers have enabled dramatic improvements in high resolution and high sensitivity chemical sensing at mid and long wave infrared wavelengths. Current state of the art sensors employ distributed feedback (DFB) QC devices to achieve stable single frequency output. Scanning of the DFB laser wavelength over a limited range can be obtained by varying the drive current, allowing high resolution scans of narrow spectral features. Somewhat broader tuning can be obtained by varying the temperature of the DFB laser, at the expense of slow tuning rates. Further improvements in chemical sensing using QC lasers would be enabled by extending the tuning range, allowing investigation of many narrow spectral lines or broad spectral features using a single laser device.

During FY05, most of the effort at PNNL involved the development and testing of a QCL external cavity that operates close to room temperature. At the start of FY05, only pulsed QCLs were available that operate at RT so that the external cavity is demonstrated for pulsed operation; however, PNNL plans to switch to a cw device in FY06 due to significant progress made under a DARPA project at PNNL. An anti-reflective (AR) coating on the front laser facet is shown to both significantly increase the tuning range and improve the fine tuning behavior of the external cavity laser. A tuning range of $400 \mathrm{~nm}(50$ $\mathrm{cm}^{-1}$ ) is obtained for the AR coated device, with continuous tuning between Fabry-Perot (FP) modes possible. PNNL also investigated developing a miniature current controller for meeting the demands for deployable QCL applications. 


\section{Contents}

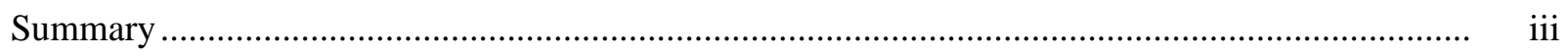

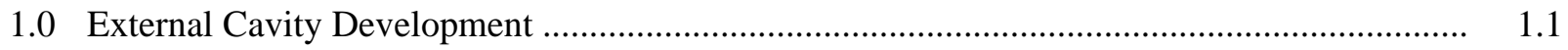

1.1 Antireflection Coating of Quantum Cascade Lasers ................................................ 1.2

1.2 Alternative Techniques for Reducing the Facet Reflectivity .............................. 1.4

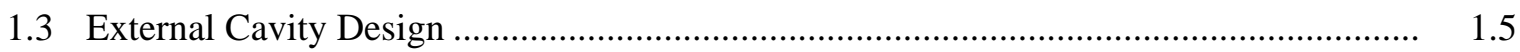

1.4 External Cavity Performance with non-AR Coated Device ................................. 1.5

1.5 External Cavity Performance with AR Coated Device .............................................. 1.7

1.6 Effects of Pulsed Operation on External Cavity Behavior ................................... 1.9

1.7 Effects of Spectral Chirp on External Cavity Behavior …............................................ 1.11

1.8 Effects of Pulsed Operation on Fine Tuning Behavior ..................................... 1.15

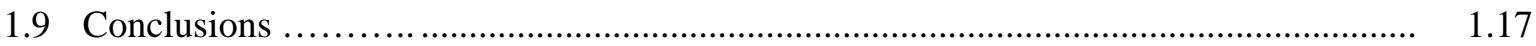

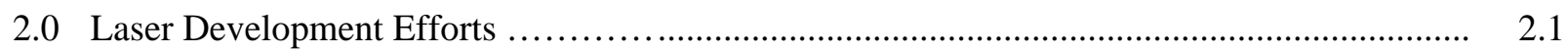

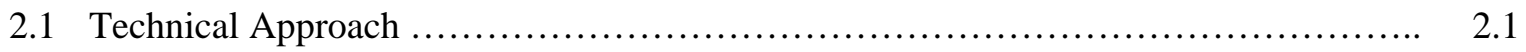

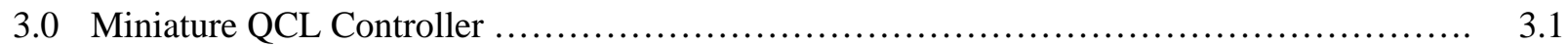




\section{Figures}

1.1 Reflectance and transmittance of witness sample for AR coating ...................................... 1.2

1.2 Images of AR coated QC device before and after thermal cycling tests ............................ 1.3

1.3 Diagram of Littman-Metcalf external cavity configuration.......................................... 1.5

1.4 Spectra of external cavity QC laser without AR coating ................................................. 1.6

1.5 Coarse tuning of external cavity QC laser without AR coating. ....................................... 1.6

1.6 Fine tuning of external cavity QC laser without AR coating ............................................ 1.7

1.7 Spectrum of AR coated QCL in external cavity ..................................................... 1.8

1.8 Coarse tuning of AR coated QCL in external cavity ......................................................... 1.8

1.9 Fine tuning of AR coated QCL in external cavity ........................................................ 1.9

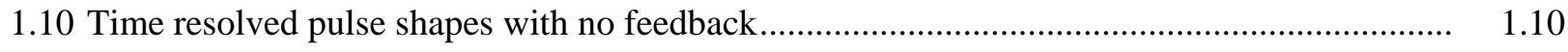

1.11 Differences in LI curves due to measurement technique................................................. 1.11

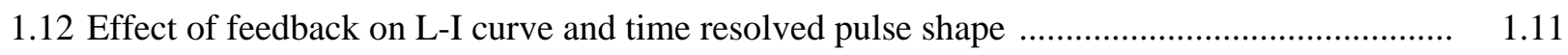

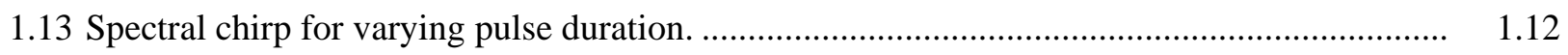

1.14 Illustration of spectral chirp during current pulse...................................................... 1.13

1.15 Output power fluctuations during pulse due to spectral chirp......................................... 1.14

1.16 Spectral chirp rate of FP modes versus peak drive current............................................... 1.14

1.17 Laser spectrum for different regions of current pulse...................................................... 1.15

1.18 Pulse shape and spectrum of external cavity laser as mirror is tuned.................................. 1.16

1.19 Linearization of fine tuning using de bias current ....................................................... 1.17 



\subsection{External Cavity Development}

One approach to extending the tuning range of QC lasers involves using an external cavity configuration. In this case, a Fabry-Perot (FP) QC laser is used, which normally lases simultaneously on many modes defined by the length and refractive index of the laser device. To make the laser output single frequency, the QC device is placed into an external cavity which includes wavelength selective optics, such as a diffraction grating, and a mechanism to optically feed back a selected wavelength to the QC gain medium. Alternately, one can view the entire system as a coupled cavity consisting of the intrinsic FP cavity of the QC laser and the external cavity. If the total optical loss at the feedback selected wavelength is made less that the loss of the intrinsic FP modes of the QC laser, the output will become single mode. On the other hand, if the loss at any or all of the FP modes is smaller, the external cavity feedback is ineffective and the laser will operate multimode. An ideal external cavity laser would allow single frequency operation over the entire gain bandwidth, where the lasing wavelength is selected by the external cavity tuning mechanism.

Research using visible and near infrared laser diodes has shown that a key component of successful external cavity laser design involves reducing the reflectivity of the laser diode facet, typically by applying an antireflective (AR) coating. The goal of the AR coating is to increase the loss of the intrinsic FP modes so that the external cavity can select the lasing wavelength. Without an AR coated device, the external cavity can only select wavelengths near FP modes, and only near the center of the gain bandwidth. Therefore, the development of high-performance AR coatings for QC lasers is essential for good external cavity laser performance.

To achieve the narrow laser linewidths required for high resolution spectroscopy, external cavity devices are typically operated in continuous wave $(\mathrm{CW})$ mode. At the time the research described here was conducted, $\mathrm{CW}$ mode QC lasers could only be operated at cryogenic temperatures. The requirement of low temperature operation poses serious challenges for AR coating efforts. In particular, because of large layer thicknesses required for infrared wavelengths, it is difficult to deposit an AR coating which survives repeated cycling to cryogenic temperatures without damage. To avoid cryogenic operation, the QC lasers can be operated near room temperature, but only in pulsed current mode. Operation of an external cavity laser in pulsed mode is not ideal, because transient heating can increase the laser wavelength due to spectral chirping effects. Note that this is also problematic for pulsed DFB devices, where extremely short pulses (or time resolved techniques) are required for high resolution spectroscopy.

This section describes research performed to characterize and study an external cavity QC laser. A central focus of this research was determining the effects of AR coating on the laser operation in an external cavity configuration. To study the effects of AR coating apart from issues of thermal cycling, the QC laser was operated near room temperature in pulsed mode. It was found that the AR coating both significantly increased the tuning range and improved the fine tuning behavior of the external cavity laser. A tuning range of $400 \mathrm{~nm}\left(50 \mathrm{~cm}^{-1}\right)$ was obtained for the AR coated device, with continuous tuning between FP modes possible. We also discuss the issues related to operating the laser in pulsed mode, including a determination of spectral chirp rates due to transient heating effects. The results indicate that despite the presence of transient heating, the external cavity output wavelength remains fixed over the 
duration of the pulse. Finally, we show how the presence of small errors in fine tuning caused by pulsed operation can be corrected.

\subsection{Antireflection coating of quantum cascade lasers}

PNNL had previously designed and deposited AR coatings for quantum cascade (QC) lasers operating at wavelengths near 8 microns consisting of a quarter wavelength layer of ZnSe followed by a quarter wavelength layer of $\mathrm{Al}_{2} \mathrm{O}_{3}$. This $\mathrm{AR}$ coating was applied to one working QC laser and two nonlasing QC devices. Figure 1.1 shows the calculated and measured reflectance and transmittance for a germanium witness sample coated at the same time as the QC lasers.
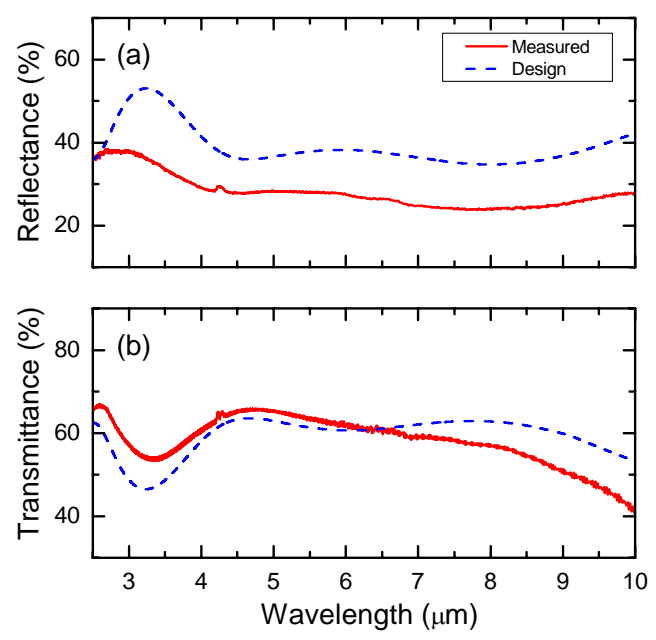

Figure. 1.1. Calculated (dashed line) and measured (solid line) reflectance and transmittance of a germanium witness sample to which the AR coating was applied.

Previous studies had indicated that similar AR coatings applied to QC laser facets did not survive thermal cycling to cryogenic temperatures, instead cracking and flaking off. However, these previous tests involved immersing the AR coated QC devices directly into liquid nitrogen followed by warming to room temperature in ambient air. While it would certainly be desirable for the AR coating to survive this highly stressful test, these conditions are not representative of the actual operating conditions of QC lasers. Therefore, we evaluated the AR coating by cooling and heating one of the nonlasing QC devices under vacuum in a standard laser dewar. In this case, the QC device was cooled and heated by conduction through metal contact with the dewar. The time to reach liquid nitrogen temperature was 15 minutes, and heating back to room temperature was accomplished in approximately one hour. Visual inspection of the laser facets under a microscope was performed to evaluate the AR coating before and after thermal cycling. 

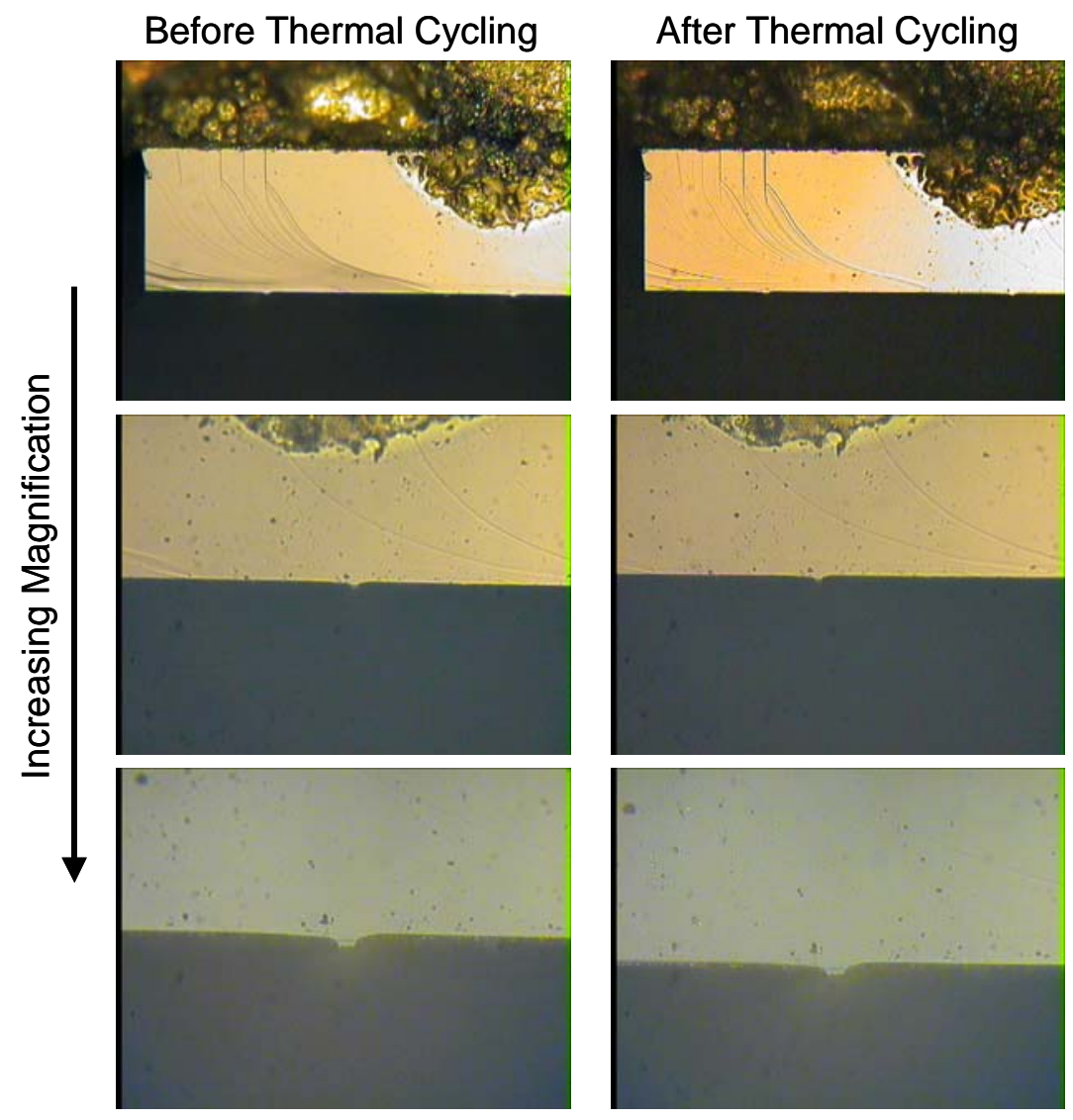

Figure 1.2. Optical microscope images of AR coated QC device facet before (left column) and after (right column) thermal cycling tests. Magnifications are 10x, 20x and 50x from top to bottom.

Figure 1.2 shows the results of the thermal cycling tests. The left column of images shows the QC laser facet for various magnifications after AR coating but before thermal cycling. The QC device is attached to a copper mount which can be seen at the top of the image, along with excess solder which has leaked onto the front facet. At low magnification, a number of steps, cracks, and defects are visible on the facet, none of which are unusual for semiconductor laser devices. At higher magnifications, the ridge waveguide is clearly visible. It should also be noted that only the quality of the AR coating near the ridge waveguide will affect the laser properties. The right column of Figure 1.2 shows images of the same regions, but after 10 complete thermal cycles. Apart from slight differences in lighting and focus, the images look very similar. There is no clear indication that the thermal cycling has damaged or degraded the AR coating by increasing the defect density.

The thermal cycling results are promising, indicating that the AR coating may in fact survive repeated cycling to cryogenic temperatures. The results cannot be considered conclusive, however. The visual inspection under a microscope is expected to discern major damage to the AR coating, but cannot verify that the operational performance of the AR coating is preserved. A more conclusive test would be to compare the properties of an AR coated, working QC laser device before and after thermal cycling. 
However, at this time only one working QC laser has been AR coated, and the risk of damaging the coating through thermal cycling remains too great.

To protect the AR coating on the working QC laser, the device was operated in pulsed mode near room temperature (TE cooled). Operating near room temperature has the additional advantages of a smaller footprint and freedom from cryogenic liquids. However, the device used could only be operated in pulsed mode near room temperature. The complications due to pulsed operation will be discussed in later sections. Nevertheless, the use of pulsed operation was acceptable for evaluating the effects of the AR coating on tuning range and fine tuning behavior.

The lasing threshold of the device for AR coating (Maxion FP-QCL-8.5-M66) was measured before and after coating. The two wire-bonded lasers on the device showed increases in lasing threshold of 5$10 \%$. The increase in lasing threshold is expected due to a decrease in facet reflectivity, and indicates that the AR coating was successful. Unfortunately, the experimental apparatus for complete characterization of the lasers was developed only after the AR coating was completed. A direct comparison of the device parameters before and after AR coating would be necessary to calculate the actual facet reflectivity after AR coating and will be performed on devices used in future coating runs.

\subsection{Alternative Techniques for Reducing the Facet Reflectivity}

In FY05, we also initiated a subcontract with Princeton University to investigate alternative techniques for reducing the facet reflectivity. They evaluated three different techniques for reducing the facet reflectivity:

1) angled-facets

2) deep-etched photonic crystal-like facets, and

3) textured facets emulating effective material quarter-wave coatings.

All three methods avoid problems with adhesion and thermal cycling and are therefore more stable and durable than conventional coatings.

Princeton estimated that the latter approach is the most efficient and practical method. In this approach, the facet surface is textured with a sub-wavelength grating using focused ion-beam milling. The sub-wavelength grating is patterned on the facet with a period that is smaller than the laser wavelength (sub-micron scale). A single quarter-wave layer with a refractive index equal to the square root of the substrate refractive index is an effective method for reducing Fresnel reflection at the surface. Thus, they have patterned facets of the QCLs by varying the fraction of material that is removed to change the effective refractive index of the semiconductor laser cavity. This pattern will have a similar effect as a quarter-wave coating and minimize surface reflections. They had not completed the work by the end of FY05 due to the late start of the contract so that the final results will be completed in FY06 to determine the effectiveness and reliability of the facet texturing approach. 


\subsection{External cavity design}

For external cavity operation, the Littman-Metcalf configuration shown in Figure 1.3 was used. This cavity configuration has the advantages of high wavelength selectivity due to the double passing of the grating, and pointing stability during tuning because the grating is fixed in position. For research and development purposes, this cavity design also has the benefit that the optical feedback can be turned off by simply blocking the mirror. The QCL was mounted in a commercial thermoelectric cooler housing designed by Alpes Laser. The output from the QCL was collimated using a custom doublet lens system with $\mathrm{NA}=0.7$. Wavelength selective feedback was provided by a 150 groove $/ \mathrm{mm}$ gold coated diffraction grating, blazed for $8.579 \mu \mathrm{m}$, and with a measured first order diffraction efficiency of $85 \%$ for polarization perpendicular to the grating grooves. The diffraction grating was oriented for near-grazing incidence, and the first-order diffracted beam was sent to a gold coated mirror. Wavelength tuning was accomplished by changing the angle of the mirror, which selected the wavelengths for feedback into the laser. The laser output was taken from the zeroth order diffracted beam off the grating. The total external cavity length was $20 \mathrm{~cm}$.

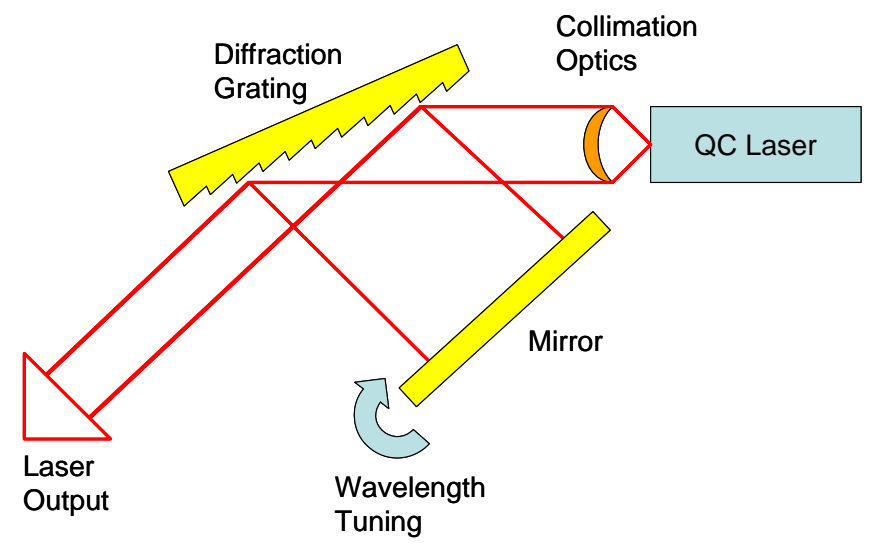

Figure 1.3. Diagram of Littman-Metcalf external cavity configuration.

\subsection{External cavity performance with non-AR coated device}

Initial evaluation of the external cavity laser was performed using a QC laser without AR coating. The device used here (Maxion FP-QCL-8.5-M62-A3) was selected for its similar properties to the device which was AR coated. The laser was operated at $10^{\circ} \mathrm{C}$, with current pulses of $100 \mathrm{~ns}$ duration at a repetition rate of $100 \mathrm{kHz}$, giving a duty cycle of $1 \%$. The measured lasing threshold was at a peak current of $1.24 \mathrm{~A}$. Using the active region area of $2.47 \times 10^{4} \mathrm{~cm}^{2}$ gives a lasing threshold peak current density of $5.0 \mathrm{kA} / \mathrm{cm}^{2}$. 
When operated without feedback, the device lased on several FP modes of the QC laser. Figure 1.4(a) shows a representative spectrum of the laser output for a peak current of 1.6 A. Spectra were obtained using a Bruker OPAG 22 FTIR with a resolution of $0.5 \mathrm{~cm}^{-1}$. The measured FP mode spacing is $0.64 \mathrm{~cm}^{-1}$.

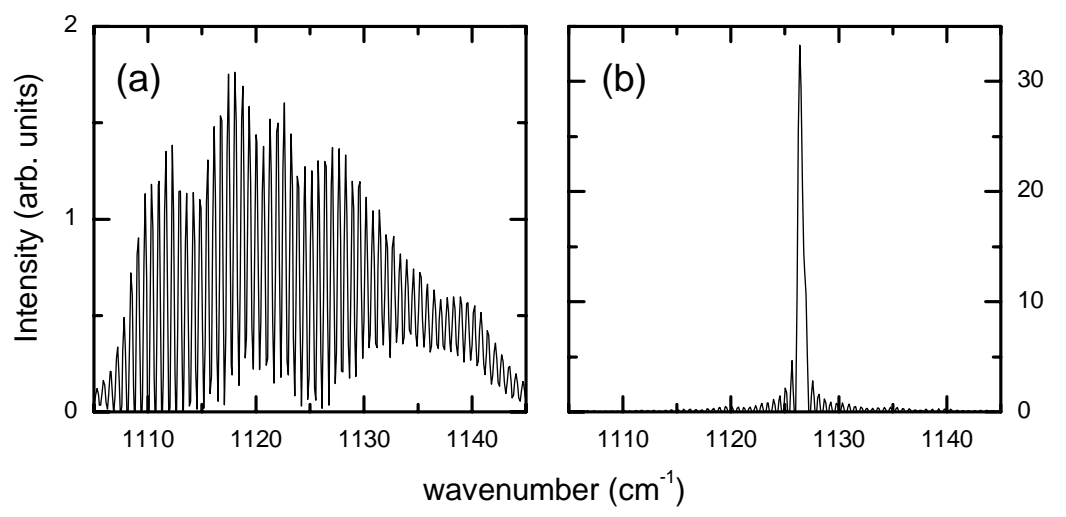

Figure 1.4. FTIR spectra of QC laser without AR coating, and (a) without feedback, (b) with feedback.

When operated with feedback, the laser output could be reduced to a narrow spectral band (below the resolution of the FTIR), as shown in Figure 1.4(b). Keeping the laser output single mode, the lasing

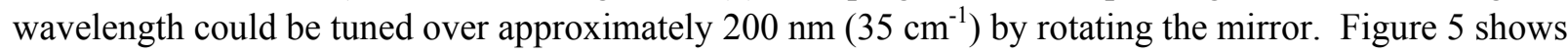
the wavelength tuning of the external cavity laser as a function of the mirror angle. Note that the output power drops significantly for wavelengths near the edges of the tuning range, and competition from lasing on the FP modes becomes more likely.

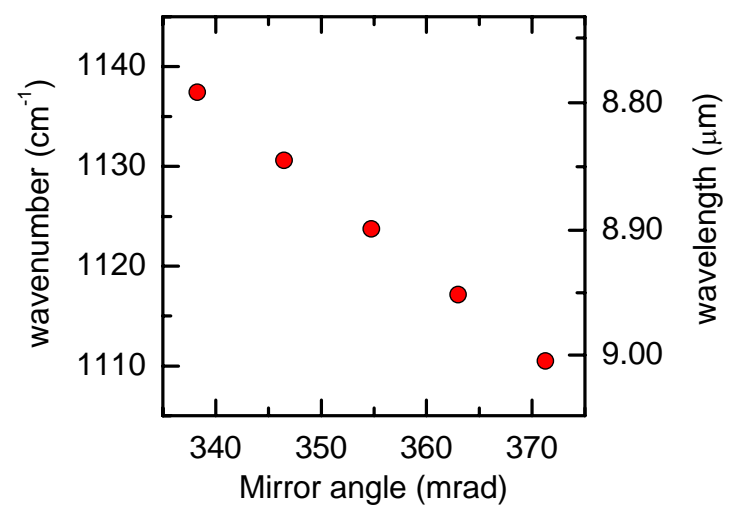

Figure 1.5. Coarse tuning of the external cavity QC laser without AR coating. The peak current was $1.8 \mathrm{~A}$.

The fine tuning behavior of the external cavity laser without AR coating is shown in Figure 1.6. It is seen from Figure 1.6(a) that the laser does not tune continuously, but instead shows linear tuning regions separated by gaps at a periodicity corresponding to the FP mode spacing. This behavior is reflected in Figure 1.6(b), which shows FTIR spectra for five successive mirror angles of Figure 1.6(a). The external 
cavity laser wavelength shows a strong tendency to remain near the FP modes and cannot be made single mode for all intermediate wavelengths.
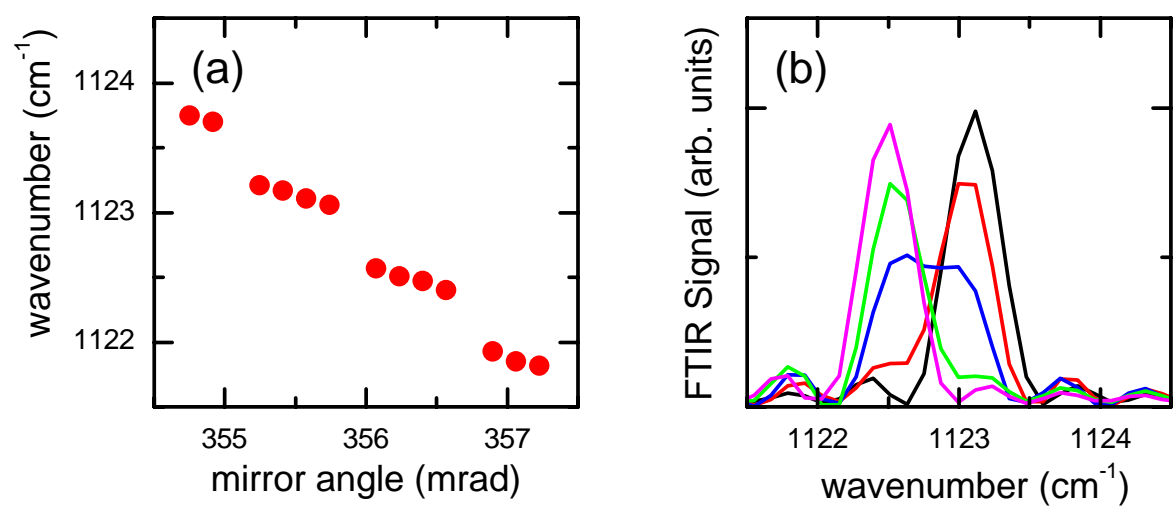

Figure 1.6. Fine tuning of $\mathrm{QC}$ laser without AR coating. (a) Wavelength variation with mirror angle.

(b) FTIR spectra for five successive mirror angles.

\subsection{External cavity performance with AR coated device}

The AR coated device was also operated at $10^{\circ} \mathrm{C}$, with current pulses of $100 \mathrm{~ns}$ duration at a repetition rate of $100 \mathrm{kHz}$ ( $1 \%$ duty cycle). Without feedback, the lasing threshold was measured to occur at 1.76 A peak current. The area of the active region is $4.01 \times 10^{-4} \mathrm{~cm}^{2}$, giving a threshold peak current density of $4.4 \mathrm{kA} / \mathrm{cm}^{2}$. Figure 1.7(a) shows the FP modes in the output spectrum at a peak current of $1.9 \mathrm{~A}$; the FP mode spacing is measured to be $0.64 \mathrm{~cm}^{-1}$.

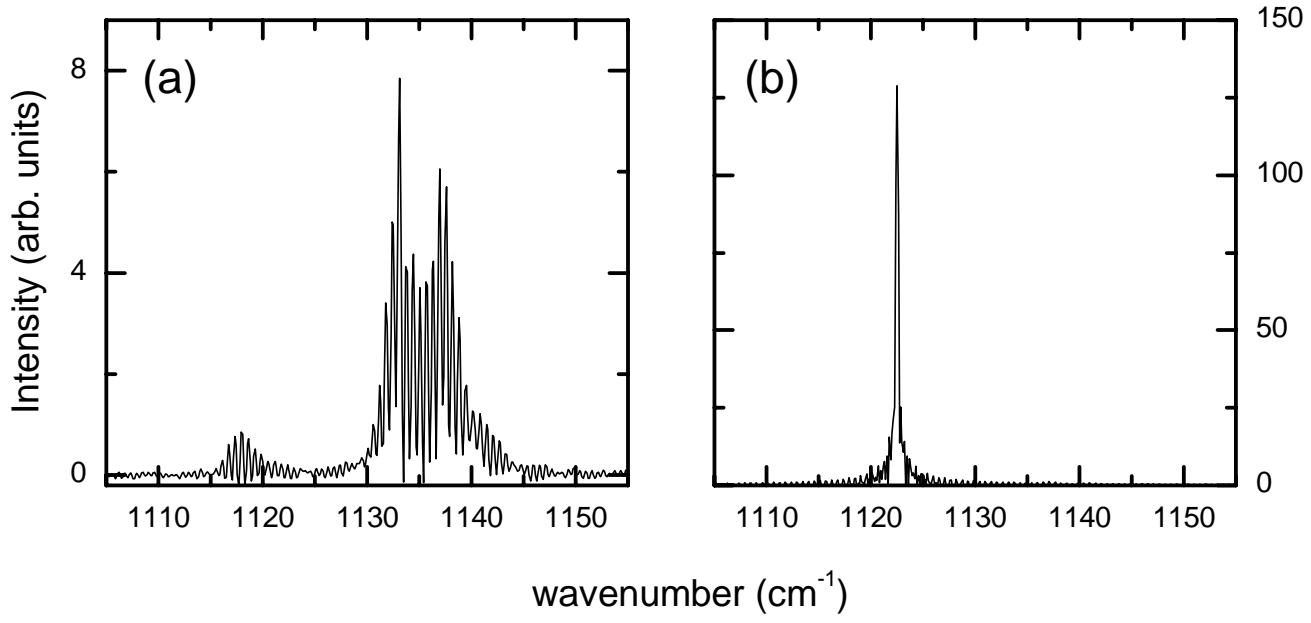

Figure 1.7. Output spectrum of AR coated QCL at a peak current of 1.9 A, (a) without feedback, and (b) with feedback. 
With feedback the laser spectrum could again be made single mode, as shown in Figure 1.7(b). The coarse tuning behavior of the AR coated QCL operated with feedback is shown in Figure 1.8 for a peak current of 2.2 A. Figure 1.8(a) shows 8 separate spectra taken over the tuning range of approximately 400 $\mathrm{nm}\left(50 \mathrm{~cm}^{-1}\right)$. The variation of wavelength with mirror angle is shown in Figure 1.8(b).
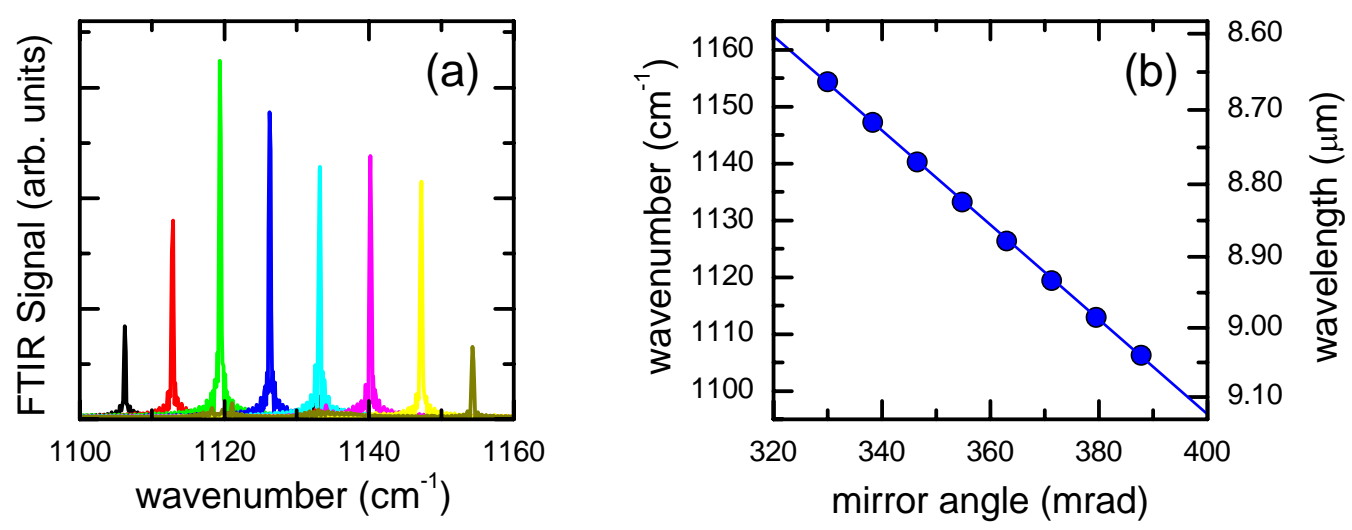

Figure 1.8. Coarse tuning of AR coated QCL at a current of $2.2 \mathrm{~A}$. (a) Spectra for 8 mirror positions distributed over tuning range. (b) Variation of wavelength with mirror angle.

The fine tuning behavior of the AR coated device is shown in Figure 1.9. Figure 1.9(a) shows the wavelength variation with mirror angle. Although a periodicity is still seen corresponding to the FP mode spacing, the fine tuning behavior is noticeably smoother than for the device without AR coating. The series of spectra in Figure 1.9(b) demonstrates a continuous tuning between FP modes; however, a residual power fluctuation is clearly present. Also note that due to the limited resolution of the FTIR, the external cavity longitudinal modes cannot be resolved (the mode spacing is calculated to be $0.025 \mathrm{~cm}^{-1}$ for the $20 \mathrm{~cm}$ cavity length). True continuous tuning between the longitudinal modes was not expected, because the cavity length was not changed in the appropriate amount relative to the mirror angle. 

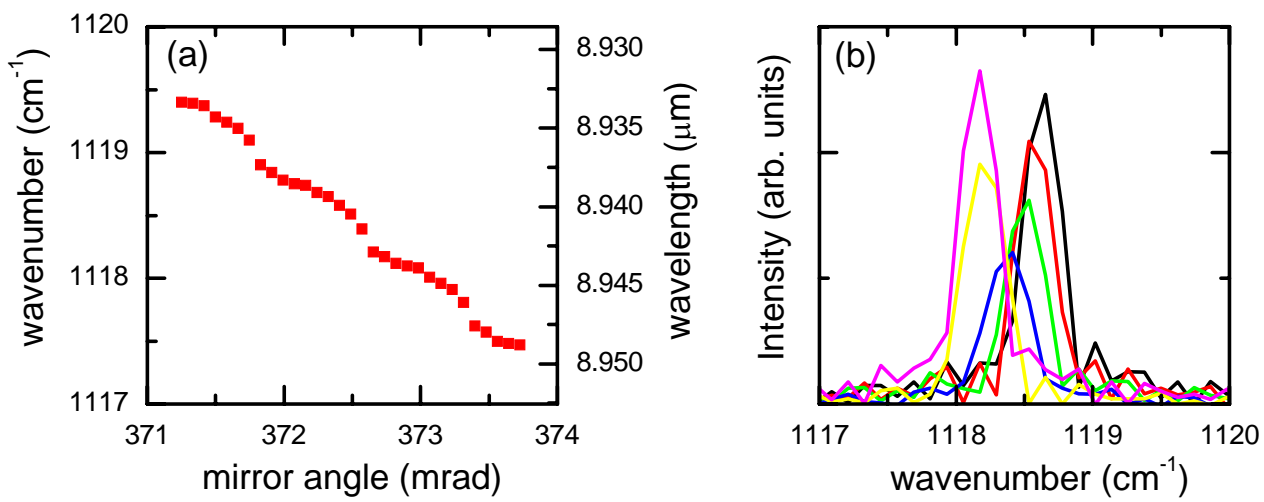

Figure 1.9. Fine tuning of $A R$ coated $Q C L$ at a current of $2.2 \mathrm{~A}$. (a) Wavelength variation with mirror angle. (b) Series of spectra as wavelength is tuned.

Although not an ideal direct comparison since different devices were used, the above results indicate that application of the AR coating has significantly improved the performance of the external cavity laser. First, the tuning range is nearly doubled for the AR coated device. Second, while the device without AR coating exhibited a strong tendency to hop between the FP modes, the AR coated device could be tuned continuously.

\subsection{Effects of pulsed operation on external cavity behavior}

As noted above, operation of the external cavity QC laser in pulsed mode leads to a number of complications not present for $\mathrm{CW}$ operation. Although $\mathrm{CW}$ operation will probably be preferred for the best external cavity device, there could also be applications for a pulsed mode version. The efforts to characterize and understand the pulsed mode behavior are described below. All results in this section are for the AR coated device.

Measuring the time-resolved pulse shapes requires temporal resolutions on nanosecond time scales. Room temperature MCZT detectors have the necessary temporal resolution, but are too noisy for accurate pulse characterization at low optical powers. Liquid nitrogen cooled photovoltaic MCT detectors have much lower noise levels, but are often not optimized for high speed measurements. We found that the best results were obtained by using a small area $(100 \mu \mathrm{m})$, liquid nitrogen cooled MCT detector (Fermionics PV-9-100), operated at a negative bias voltage of $100 \mathrm{mV}$. The bias voltage was found to be essential to obtain the temporal resolution required and also to improve the linearity of the detector. Signals were recorded directly on a high speed oscilloscope, eliminating the need for additional high speed preamplification stages. Temporal resolutions better than $5 \mathrm{~ns}$ could be obtained using this detection scheme. Multiple traces (typically 256) were averaged to reduce noise levels. 
Examples of time resolved output pulses from the laser operated without feedback are shown in Figure 1.10 for two different injection currents. At the beginning of the current pulse, a large peak in the optical power is observed. After this initial transient peak, the optical power is reduced to a roughly constant level at which it remains for most of the current pulse. Another transient peak in optical power can be seen at the end of the current pulse. The origin of the transient peaks is unknown, but it could be caused by the shape of the current pulse itself, ringing effects when the current is switched, or transient heating effects within the QC laser active region. Regardless of the cause of the transient peaks, their effect cannot be ignored. For example, Figure 1.11 shows the L-I curve measured in two ways. Figure 1.11(a) shows the curve obtained by measuring the total integrated optical power. Figure 1.11(b) shows the curve obtained by measuring the average optical power for the central nearly constant region of the pulse. When the transient peaks are excluded, the L-I curve exhibits a much sharper and well-defined lasing threshold corresponding to the onset of lasing for the central region of the pulse. For the purpose of predicting the behavior of a CW external cavity QC laser, we can largely ignore the transient peaks and focus on the central region of the pulse. We will therefore use the central region of the pulse to define the lasing threshold.

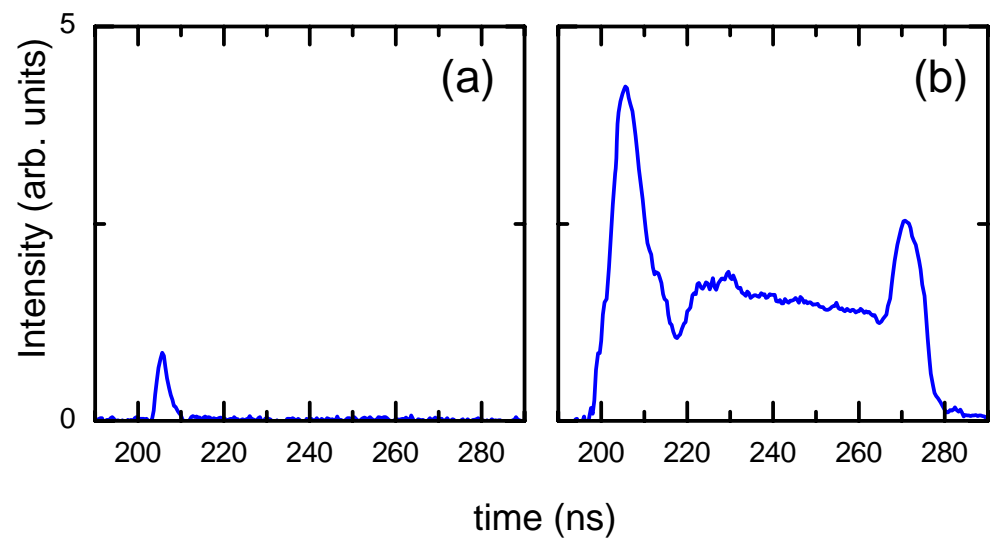

Figure 1.10. Time resolved pulse shape (no feedback) for peak currents of (a) $1.5 \mathrm{~A}$, and (b) $2.0 \mathrm{~A}$. 

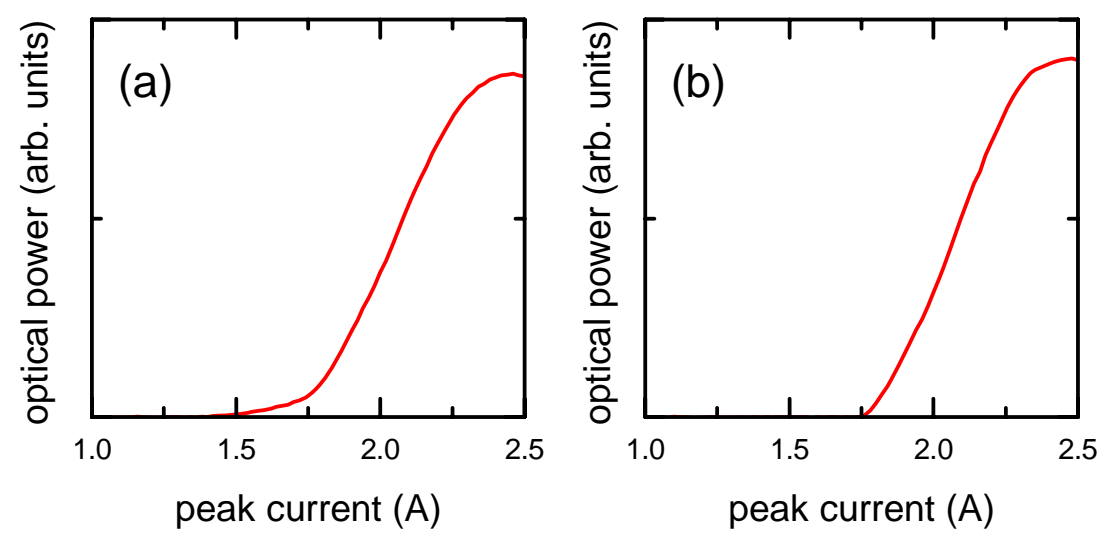

Figure 1.11. L-I curves obtained by measuring (a) integrated optical power, and (b) average optical power for central region of pulse. The laser was operated without feedback.

For the external cavity laser, the effects of feedback on the L-I curve and pulse shape are shown in Figure 1.12. Figure 1.12(a) shows the reduction in lasing threshold in the presence of feedback, while Figure 1.12(b) shows the change in pulse shape for a peak current of $1.7 \mathrm{~A}$. While the $1.7 \mathrm{~A}$ peak current is below the lasing threshold without feedback, the presence of feedback reduces the cavity loss enough to allow the device to lase over the duration of the current pulse. Note that the effects of feedback on the initial transient peak are much smaller than on the central region of the current pulse.
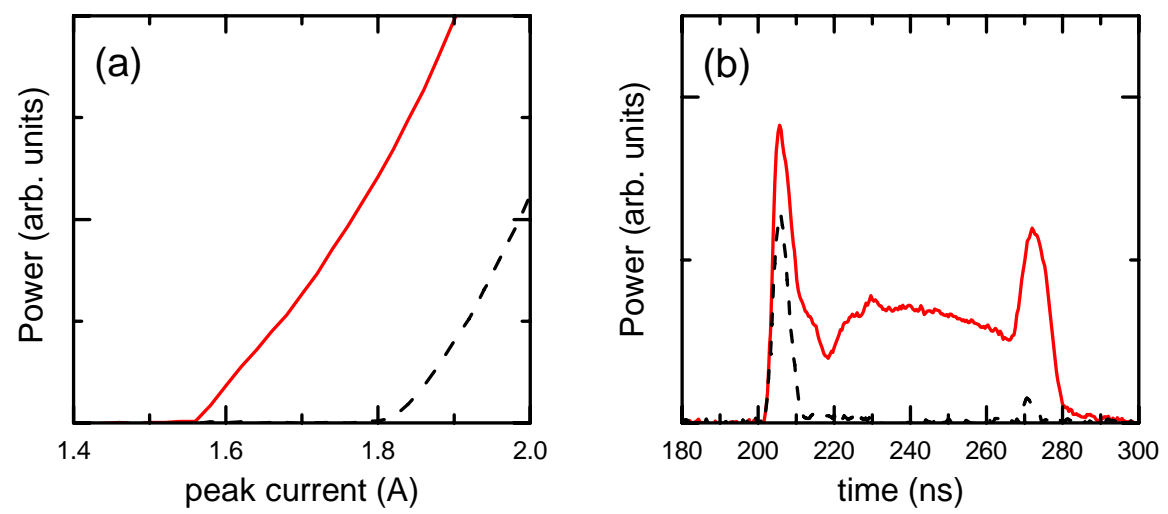

Figure 1.12. Effect of feedback on (a) L-I curve, and (b) time resolved pulse shape at 1.7 A peak current. The dashed line is data without feedback, and the solid line is with feedback.

\subsection{Effects of spectral chirp on external cavity behavior}

It is well known that the wavelength of both FP and DFB QC lasers shifts with temperature; furthermore, this effect is also present due to transient heating effects during an individual current pulse. 
It is important to understand the effects of this spectral chirp during the current pulse on the external cavity laser. To study this behavior, we first varied the pulse duration while keeping the peak current constant. Figure 1.13 shows time resolved output pulses and spectra as the pulse duration was changed from $50 \mathrm{~ns}$ to $200 \mathrm{~ns}$ while keeping the peak current fixed at $2.3 \mathrm{~A}$. Without feedback, shown in Figure 1.13(a) and 1.13(b), the longer pulse durations lead to a red-shift in the FP modes due to heating of the active region. The apparent broadening of the peaks in Figure 1.13(b) also indicates that the shift is continuous. With feedback, as shown in Figure 1.13(c) and 1.13(d), the spectrum shows no shift or broadening as the pulse duration was changed. Instead, the pulse shape showed an oscillatory power fluctuation in time.

Since the applied current pulse is identical with or without feedback, the resulting active region heating will also be the same. We can further conclude that the FP mode shift seen in Figure 1.13(b) will also occur when feedback is present. The feedback mechanism, however, acts to select one particular lasing wavelength, independent of time. As shown above in Figure 1.9(b), the output power shows a strong dependence on the position of the feedback selected wavelength relative to the FP modes. Therefore, the overall result is that as the FP modes shift across the feedback selected wavelength, this spectral shift is converted into the power variation shown in Figure 1.13(c).
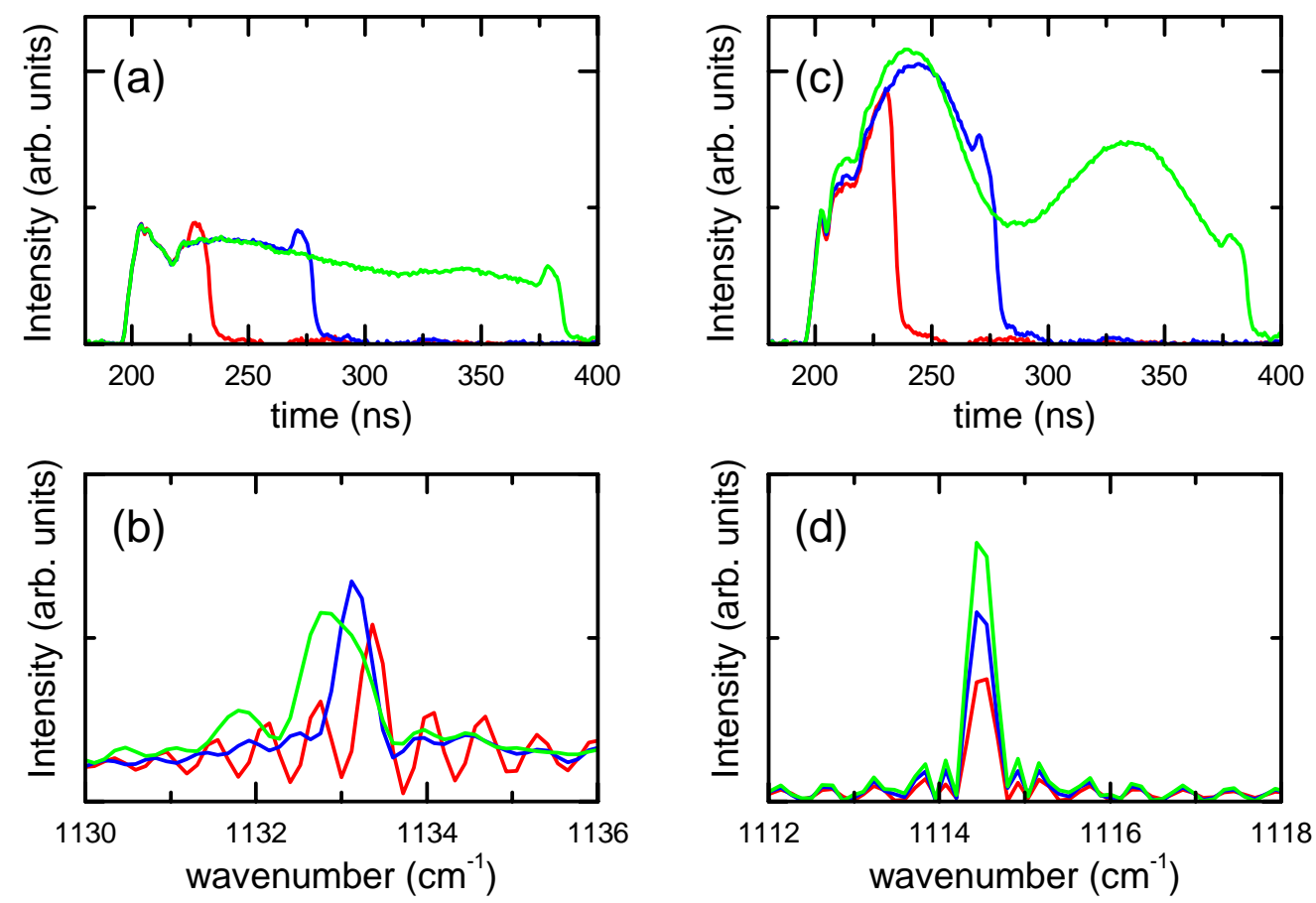

Figure 1.13. Effects of varying pulse duration at constant peak current of $2.3 \mathrm{~A}$. The pulse shape (top) and spectrum (bottom) are shown for the laser operating without feedback (a)-(b), and with feedback (c)-(d).

Figure 1.14(a) illustrates the behavior observed in Figure 1.13. The solid red line shows the feedback selected mode, which has a constant wavelength over time. The dashed lines show the FP modes, which red-shift over the duration of the current pulse (here the shift is assumed to be linear in time). As the two 
FP modes in blue scan across the feedback selected wavelength, the output power fluctuates, as shown in Figure 1.14(b). Note that this is intended only to be a simple illustration based on the experimental observations. A full theoretical treatment of the effects of spectral chirp in an external cavity QC laser is beyond the scope of this report.
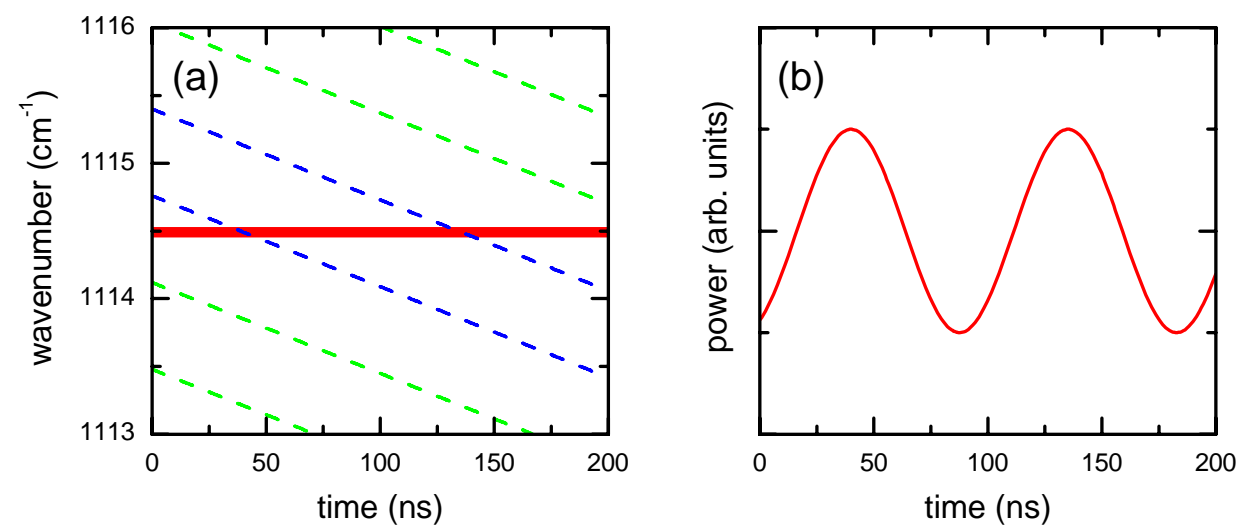

Figure 1.14. Illustration of spectral chirp during current pulse. (a) Spectral position versus time.

The solid red line shows the feedback selected wavelength, while the dashed lines show the FP modes which shift in wavelength. (b) Output power versus time.

The above experiment and analysis can also be used to determine values of the spectral chirp. Figure 1.15 shows the output power oscillations for four drive currents. As expected, the rate of spectral chirp depends on the drive current, indicated by the change in the period of output power oscillations. Using the oscillation periods measured in Figure 1.15, and knowing that the FP modes are separated by 0.64 $\mathrm{cm}^{-1}$, we can calculate the rates of spectral chirp. The result is shown in Figure 1.16, where the spectral chirp rates are plotted versus peak drive current. Note that these results assume that the spectral shifts are linear in time and that the FP mode spacing does not change. 


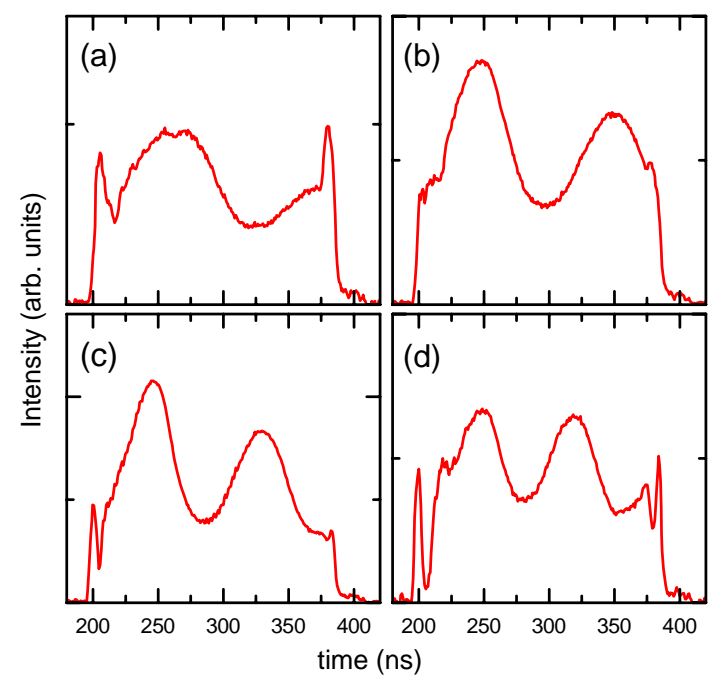

Figure 1.15. Output power fluctuations for peak currents of (a) $2.2 \mathrm{~A}$, (b) $2.5 \mathrm{~A}$, (c) $2.8 \mathrm{~A}$, (d) $3.1 \mathrm{~A}$.

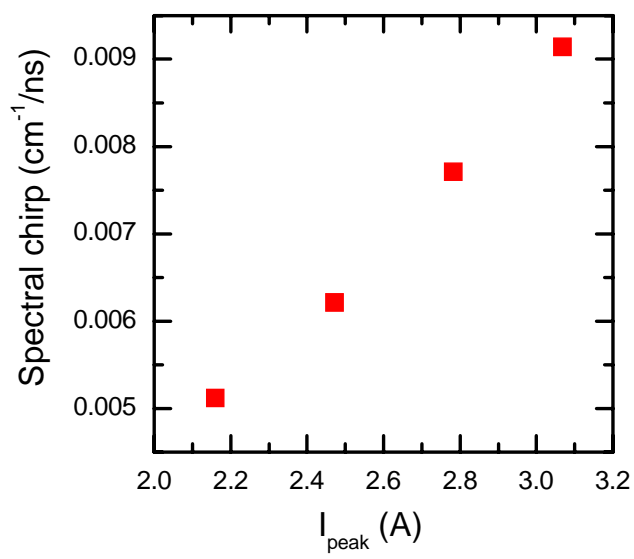

Figure 1.16. Spectral chirp rate of FP modes versus peak drive current determined from results of Figure 1.15.

We have observed that the external cavity laser output remains fixed in wavelength over the duration of the pulse; however, there can still be residual contributions to the spectrum at the FP modes. Figure 1.17(a) shows the external cavity laser spectrum on a logarithmic scale for a $50 \mathrm{~ns}$ current pulse at a peak current of 2.3 A. Output from the FP modes is clearly visible in the spectrum. We can remove the effects from the transient peak at the beginning of the pulse, by subtracting the spectrum for the $50 \mathrm{~ns}$ pulse from the spectrum for a $200 \mathrm{~ns}$ pulse. The results of this subtraction are shown in Figure 1.17(b). Here it is seen that the FP modes are not present, indicating that they contributed to the spectrum during the beginning of the pulse. We can also estimate a side-mode suppression ratio (SMSR) greater than $25 \mathrm{~dB}$ 
from the spectrum in Figure 1.17(b). Note, however, that the low resolution of the FTIR, and the broad pedestal in the spectrum (due at least in part to instrumental artifacts) preclude a determination of the true SMSR.

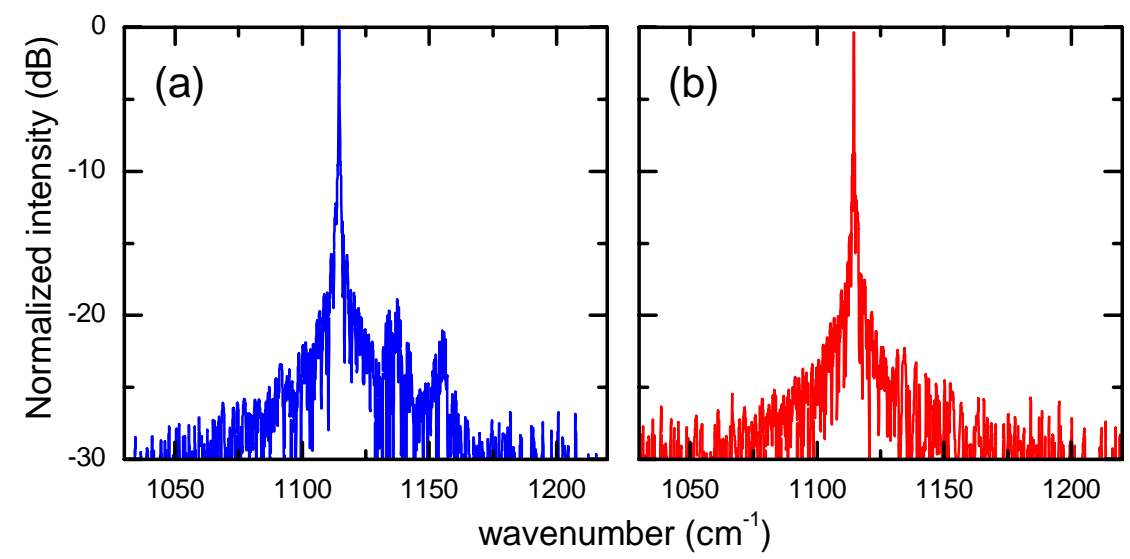

Figure 1.17. Normalized external cavity laser spectrum for different regions of current pulse with $2.3 \mathrm{~A}$ peak current. (a) Spectrum for $50 \mathrm{~ns}$ pulse. (b) Difference spectrum for $200 \mathrm{~ns}$ and $50 \mathrm{~ns}$ pulse.

As noted before, the highest performance of external cavity lasers is typically obtained in CW operation. However, the results here show that despite the spectral chirp of the FP modes present during pulsed operation, the external cavity output wavelength remains fixed over the duration of the pulse. Furthermore, under optimal feedback conditions, undesirable contributions to the laser output at the FP modes occur primarily at the start of the current pulse. This behavior indicates that a pulsed external cavity QC laser could be a viable option for a mid- or high-resolution spectroscopic system. The output power fluctuations over the pulse duration would not be problematic for applications where the integrated power was detected. The primary limitations would arise from the transient effects at the beginning and end of the current pulse. Proper shaping of the current pulse could be used to improve operation in this case. Further studies of the linewidth and tuning properties of the pulsed laser at higher spectral resolution are needed to fully evaluate its performance.

\subsection{Effects of pulsed operation of fine tuning behavior}

Operation in pulsed mode was also found to have interesting effects on the fine tuning behavior of the external cavity laser, which can be traced to the spectral chirp of the FP modes. It was found that as the mirror angle was tuned, both the pulse shape and output spectrum underwent changes periodic with the FP mode spacing. For certain mirror angles, the spectrum exhibited good single mode output, while for others residual FP modes were present in the output. Figures 1.18(a) and 1.18(b) show an example of the pulse shape and spectrum respectively for good single mode output. The pulse shape shows a large intensity increase over the case with no feedback, and the spectrum contains only small contributions from the FP modes. Figures 1.18(c) and 1.18(d) show the output for a different mirror angle. Here the pulse shape contains a region of low intensity and there is a large contribution of FP modes in the output spectrum. For this mirror angle, there is a window of time wherein the feedback selected wavelength has 
higher loss than the FP modes. It was seen above that the spectral chirp of the FP modes leads to power fluctuations in output power. The behavior seen here indicates that the relative spectral position of the grating selected wavelength and the FP modes at the start of the pulse also has a strong effect on the laser output.

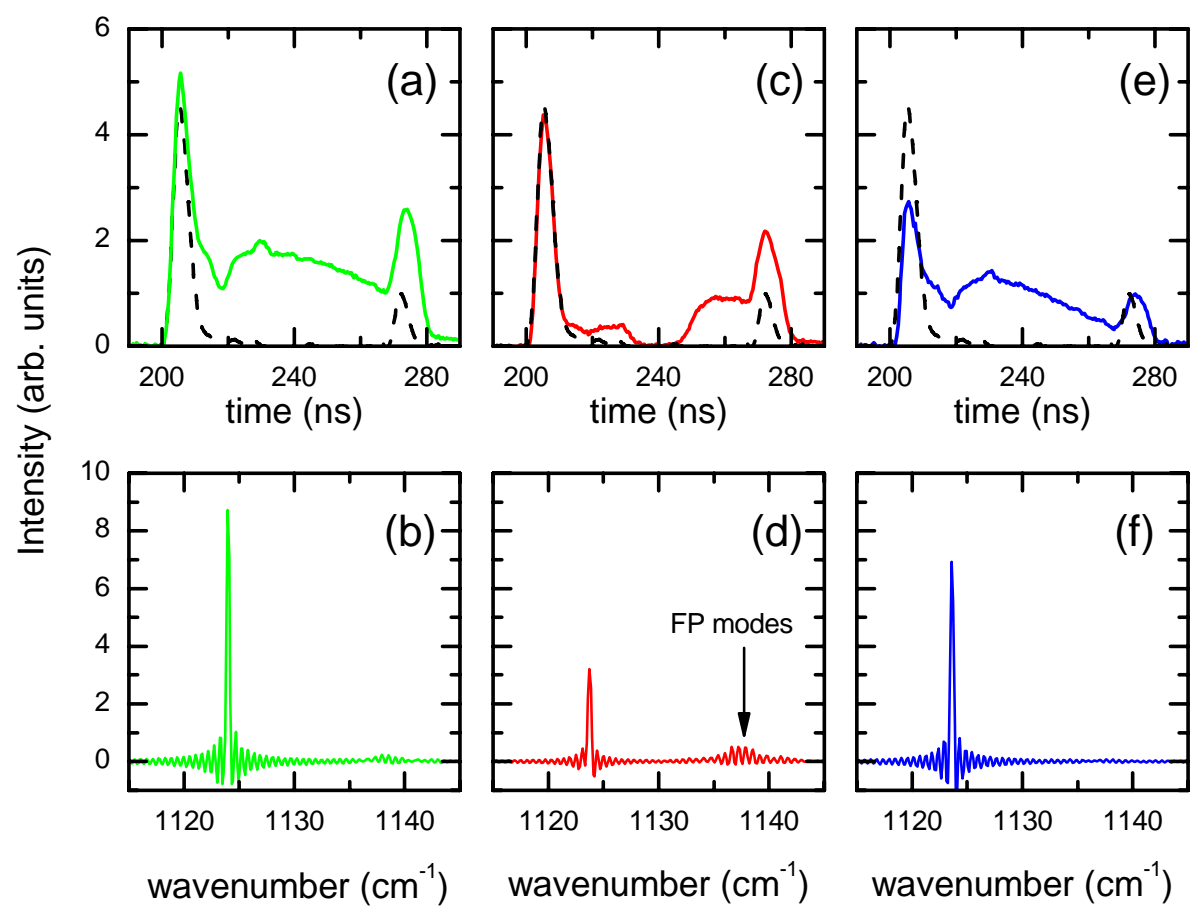

Figure 1.18. Pulse shape (top) and output spectrum (bottom) of external cavity laser as mirror angle is tuned. (a) and (b) show the output for optimal feedback conditions. (c) and (d) show the output for less favorable feedback conditions. (e) and (f) show the output for the same mirror angle as (c) and (d) but with an applied dc bias current of $107 \mathrm{~mA}$. For all cases, the peak current was $1.7 \mathrm{~A}$.

Therefore, in order to improve the fine tuning of the laser, a mechanism is needed to control the relative starting positions of the FP modes and the feedback selected wavelength. This control can be obtained by applying a dc bias current to the QC laser. The dc bias current heats the active region and can be used to shift the position of the FP modes at the start of the current pulse. Figures 1.18(e) and (f) show the laser output for the same mirror angle as Figures 1.18(c) and 1.18(d), but with a $107 \mathrm{~mA}$ dc bias current applied to the QC laser. This bias current was selected by adjusting its value until the pulse shape matched the one shown in Figure 1.18(a). When this condition was met, the spectrum returned to single mode output without the presence of the FP modes. An additional effect of the bias current was to reduce the output power, which is expected due to the increased temperature of the active region. 


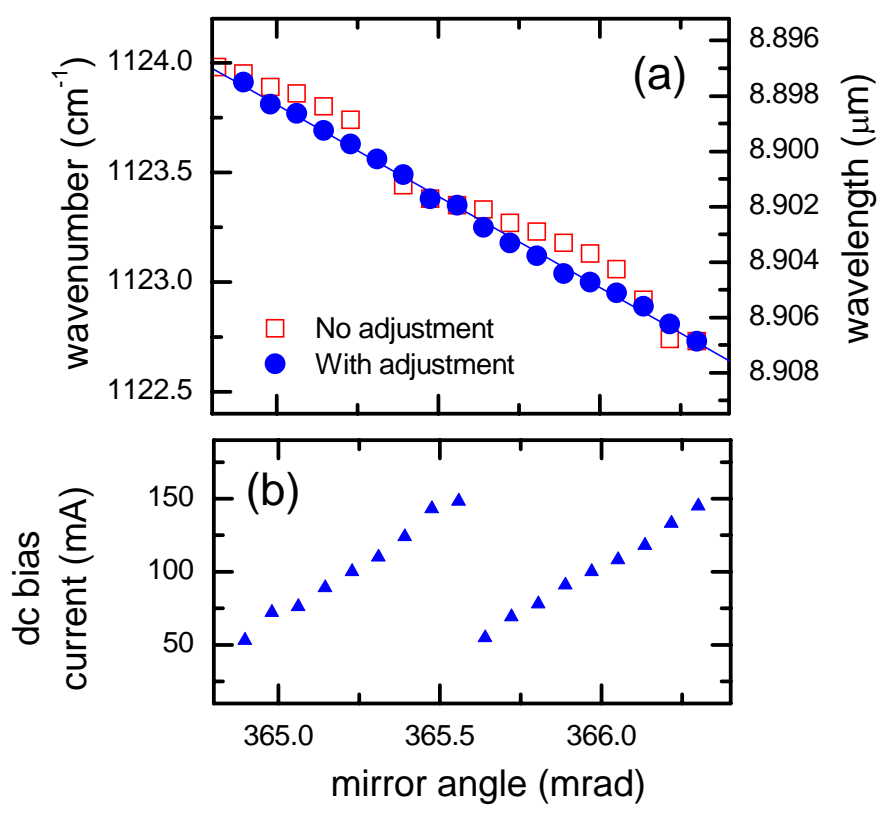

Figure 1.19. Linearization of fine tuning using dc bias current. (a) Output wavelength versus mirror angle with (circles) and without (squares) dc bias current. (b) Value of dc bias current applied as function of mirror angle. The peak pulsed current was $1.7 \mathrm{~A}$.

Figure 1.19(a) shows the improvement in fine tuning behavior when the bias current was applied and set using the same procedure described above. The tuning with bias current applied (circles) demonstrates a higher linearity than the tuning without bias current (squares). The optimized values of the dc bias current are shown in Figure 1.19(b).

\subsection{Conclusions}

In summary, we have assembled and characterized an external cavity QC laser operating near room temperature in pulsed mode. When operated using a QC device without AR coating, the external cavity laser could be tuned over $200 \mathrm{~nm}\left(35 \mathrm{~cm}^{-1}\right)$, but could not be tuned continuously between the FP modes of the QC laser. The performance of the external cavity laser was improved through the application of an $\mathrm{AR}$ coating to the QC device, which increased the tuning range to $400 \mathrm{~nm}\left(50 \mathrm{~cm}^{-1}\right)$. Furthermore, the wavelength could be tuned continuously between the FP modes.

The effects of pulsed mode operation on the external cavity laser were also studied. It was found that despite the spectral chirp of the FP modes due to transient heating during a current pulse, the output wavelength of the external cavity laser remained fixed over the duration of the pulse. Instead, the wavelength shift was converted to a power fluctuation during the pulse. For certain mirror positions, the spectral chirp was found to result in undesirable lasing on FP modes during portions of the pulse. This behavior was corrected through the application of a dc bias current, resulting in a steady-state heating effect, and enabling more linear fine tuning. Though not directly investigated, the application of a dc bias 
current could also be used to improve the fine tuning behavior of non-AR coated QC lasers (although it would not improve the overall tuning range).

The research presented here verifies that AR coating of the QC laser has many beneficial effects on external cavity laser operation. While future research could potentially develop an AR coating useable at cryogenic temperatures, such work is probably not warranted at this time. There is currently a strong effort among QC laser manufacturers to develop room temperature $\mathrm{CW}$ lasers, and these devices would be ideal for external cavity devices. Furthermore, the results here indicate that a pulsed mode external cavity QC laser may be useful in spectroscopic applications as well, especially for applications requiring high peak power. Future work will focus on characterizing the linewidth and fine tuning behavior of pulsed QC lasers at higher spectral resolution. The research performed to study and develop pulsed external cavity QC lasers will also be directly applicable to CW devices when they become available. 


\subsection{Laser Development Efforts}

Under a complementary DARPA project, PNNL is collaborating with both Maxion Technologies, Inc. and Princeton University to develop high power (HP), room-temperature FP-QCLs. Although this project is funded by DARPA, PNNL can leverage off the successes of this project to obtain FP QCLs that operate $\mathrm{cw}$ at room temperature (RT) for the external cavity development work. A brief summary of their accomplishments from FY05 are presented in this report.

\subsection{Technical Approach}

Three main factors are important for obtaining high cw output powers at RT. First, high quality laser material is absolutely crucial for high performance. Thus, for the first three months of the DARPA project, Maxion spent time on improving the material quality by fixing problems with the MBE machine and optimizing the growth conditions until they grew high quality laser material. The other two essential factors are improving the active and waveguide designs to minimize the losses and improving the thermal management to minimize the temperature in the active region. Thus, Maxion and Princeton pursued the following objectives to achieve HP, RT performance:

1) Improved active designs for a longer upper state lifetime and better transport through injector.

2) InP top cladding for improved heat conduction through cladding (InP has a higher thermal conducitivity than InAlAs, which is the standard top cladding).

3) Improved doping profiles to lower optical losses in cladding and to reduce absorption in active region.

4) Epi-down mounting and thick Au epi-up mounting for improved overall heat conduction.

They clearly showed improvements in the maximum operating temperature by revising the active designs. The standard Lucent design is the $8.5 \mu \mathrm{m}$ design that Maxion tried to push to high temperature operation under the Alignment Lasers project. Even with an InP top cladding and thick gold to reduce the thermal resistance, the maximum operating temperature achieved with this standard Lucent design is only about $200 \mathrm{~K}$. With the double phonon design, Maxion was able to obtain a maximum operating temperature of $235 \mathrm{~K}$ using both thick gold and an InP top cladding. These results, however, are still shy of cw, RT operation.

Another approach that Maxion investigated is dry-etching of the laser ridges. Generally, the wafers are processed into a ridge waveguide by simply using wet-etching techniques. Dry etching, however, offers a more uniform approach that can produces ridges with vertical sidewalls During FY05, Maxion was able to achieve very smooth sidewalls with slopes of $\sim 62^{\circ}$. They have achieved very encouraging results with dry-etched devices along with the double phonon resonance design, for a maximum cw operating temperature of $269.3 \mathrm{~K}$ has been achieved by applying thick gold and an InP top cladding. Improvements can still be made by using a high reflective (HR) coating to increase the output power and 
lower the threshold and epi-down mounting to improve the heat dissipation. These efforts will be pursued under DARPA in FY06.

These measurements illustrate that Maxion is very close to achieving RT, cw operation. With an effective HR coating along with high-quality epi-down mounting, the lasers should provide sufficient output power at RT. PNNL plans to acquire one of these lasers for this project to modify the external cavity for cw operation at RT. 


\subsection{Miniature QCL Controller}

During FY05, preliminary work on a miniature controller for deployable QCL applications was undertaken. Typically, high performance QCL controllers are of the continuous or "linear" type, in which filtered DC power is regulated via a series pass transistor element to produce a constant current, which in turn drives the laser or QCL. In order to obtain low noise, agility and long-term stability, the efficiency of such a unit can be as low as 30 to $40 \%$. The resulting internal heat dissipation results in a minimum necessary size for such a current controller.

The alternative to linear power supplies is that of switch-mode supplies. Here, the series pass transistor operates by abruptly changing from fully on, to completely off many thousands to several millions of times a second. Filters downstream of this element in essence average the output of the series pass transistor, to produce smooth DC power. In contrast to the linear power supply, the switch-mode supply dissipates practically no power in the series pass element, since neither the on- nor the off-state dissipate significant power. In fact, typically, the switching elements don't even require heatsinks.

Drawbacks of the switch-mode power supply lie in the RF noise produced by the switching action of the series pass transistor, which can be at frequencies of many $\mathrm{MHz}$, and the imperfect nature of filters both prior to and after the series switching transistor in the circuit. During FY05, several different switch-mode architectures were investigated, with the goal of finding one that was easy to design and build, which exhibited a lack of internal resonance behavior and good control stability, while allowing the RF noise generation inherent to switch-mode units to be minimized. Ultimately, after building several prototypes, the simplest architecture of a single series pass transistor with both pre- and post-filtering was chosen.

Techniques were employed to alleviate both the issues of noise generation and compromised filtering mentioned above. A relatively high switching frequency of $1 \mathrm{MHz}$ was chosen, allowing filter components to be designed as small as practicable. Careful filter design was conducted, preventing resonances or instabilities and providing optimal filtering at all foreseeable load conditions. Special core materials for key filter inductors were chosen to allow good reactive response at the switching frequency with low losses, but poor characteristics (resulting in high losses) at much higher frequencies where radiated noise could be problematic. In this manner, such filters exhibit not only good power transfer characteristics, but also high frequency noise attenuation of the many harmonics of the switching frequency often prolifically generated by an abruptly switching transistor element.

Reduction of emitted RF noise was further effectuated by softening the switching edges of the series pass transistor, for the price of an imperceptible loss in efficiency. Preliminary investigation demonstrates superb control characteristics necessary for reliable feedback control, and very little visible noise on oscilloscope traces compared to typical switch-mode supplies, although precise measurements have yet to be taken. 
The second stage of development for this miniature QCL controller will be to incorporate an optimized switch-mode unit as a front end to a two-stage unit, the second stage being a traditional linear series pass current controller. In this way, control characteristics will be optimized, and noise generation reduced still further via the clean regulation characteristics of the linear second stage, while keeping the power dissipation in this latter stage to a bare minimum. Using these techniques, the minimum possible physical footprint for such a current controller should be achievable, while still maintaining absolute stability, and acceptable noise levels. 


\section{Distribution}

No. of

Copies

\section{OFFSITE}

LTC Ariel Cuadrado

United States DOE

NNSA/NA-22

1000 Independence Ave. SW

Washington, DC 20585

Dr. Rhys M. Williams

United States DOE

NNSA/NA-22

1000 Independence Ave. SW

Washington, DC 20585

Dr. David Berry

United States DOE

NNSA/NA-22

1000 Independence Ave. SW

Washington, DC 20585

Mr. Ralph Hastings

United States DOE

NNSA/NA-22

1000 Independence Ave. SW

Washington, DC 20585

Mr. Eric Sander

United States DOE

NNSA/NA-22

1000 Independence Ave. SW

Washington, DC 20585

Mr. W. Randy Bell

United States DOE

NNSA/NA-22

1000 Independence Ave. SW

Washington, DC 20585
No. of

Copies

Professor Henryk Temkin

Program Manager

DARPA, MTO

3701 N. Fairfax Dr.

Arlington, VA 22203-1714

\section{ONSITE}

\section{Pacific Northwest National Laboratory}

Bruckner-Lea, C K5-25

Clemmer, RG K8-29

Dudder, GB K8-29

Myers, TL K5-25

Phillips, MC K5-25

Schultz, JF (10) K5-25

Sharpe, SW K8-88

Taubman, MS K5-25

Information Release Office (7) K1-06

Distr.1 
